\title{
EVOLUINDO UMA FERRAMENTA PARA AUXILIAR O PROCESSO DE RELEASE EM AMBIENTE DE DESENVOL VIMENTO DISTRIBUÍDO DE SOFTWARE
}

\author{
Hualter O. Barbosa, Bruno A. Bonifacio e Francinildo B. Dantas \\ Sidia Instituto de Ciência e Tecnologia \\ Av. Darcy Vargas, 654 - Parque 10 de Novembro, Manaus - AM, Brasil
}

\begin{abstract}
RESUMO
O processo de globalização e novos negócios transformaram a dinâmica de desenvolvimento de software. Para atender as novas demandas, a indústria de software adaptou-se a novas metodologias que permitem diminuir os ciclos de desenvolvimento para garantir maior competitividade. Diante desse cenário o Desenvolvimento Distribuído de Software (DDS) tem se tornado elemento estratégico para o sucesso de novos produtos. No entanto, a confiabilidade, oportunidade e valor percebido pelos usuários pode ser influenciado pela automação de etapas das atividades do processo de desenvolvimento. Neste sentido, o desenvolvimento de novas tecnologias pode auxiliar desenvolvedores e gestores a melhorar a qualidade do desenvolvimento. O presente artigo apresenta um relato de experiência sobre o aprimoramento de uma das atividades processo de releases da área de produtos mobile de um Instituto de P\&D utilizando tecnologia de software. O objetivo do artigo é apresentar o aprimoramento da ferramenta CLCATCH desenvolvida com base em estudos experimentais e uma análise qualitativa sobre os pontos de melhoria para o processo de releases em projetos de atualização do Android dos dispositivos móveis. Os resultados mostram melhoria para nova versão e abordagem da CLCATCH, com pontos que podem facilitar a aplicação de novas funcionalidades da tecnologia proposta.
\end{abstract}

\section{PALAVRAS-CHAVE}

DDS, Relatório de Experiência, Melhoria de Processo, Atualização do Android

\section{INTRODUÇÃO}

As rápidas mudanças de mercado e a globalização dos negócios têm sido fortemente influenciadas por fatores externos e internos do processo de desenvolvimento de software. Diante desse cenário, os ciclos de desenvolvimento de produto se tornaram cada vez mais rápidos, para atender as demandas e para se manter competitivo frente à concorrência. À medida que o mercado de software se desenvolve, novos desafios surgem, e o principal desafio na área de engenharia de software nas últimas duas décadas têm sido a melhoria da qualidade e a redução de custo do software produzido, especialmente em ambiente de desenvolvimento distribuído de software (Audy e Prikladnick, 2007; Lima et al. 2016).

No processo de desenvolvimento cada vez mais globalizado, a confiabilidade, oportunidade e valor percebido pode ser influenciado substancialmente associados a automatização de etapas das atividades do processo de desenvolvimento. Neste sentido, o desenvolvimento de novas tecnologias pode auxiliar desenvolvedores e gestores a melhorar a qualidade dos produtos. Assim, o uso de automação de atividades do processo pode maximizar recursos e produtividade com maior eficiência (Barbosa et al. 2019).

Diante desse cenário, preocupadas com as atividades que podem causar impacto negativo na qualidade da entrega, empresas de software tem buscado soluções para otimizar seus processos utilizando tecnologias de software para automatizar atividades. O Sidia é um instituto de P\&D que tem buscado otimizar o processo de desenvolvimento de softwares para dispositivos móveis utilizando novas tecnologias de software. Neste artigo apresentamos um relato de experiência no processo de aprimoramento de novas tecnologias com a ferramenta chamada CLCATCH, por meio de estudos experimentais. O objetivo é compartilhar os resultados dos estudos feita por meio de análise qualitativa para melhoria da abordagem. A CLCATCH é uma ferramenta que foi desenvolvida em outra plataforma tecnológica para oferecer suporte necessário para alcançar maior produtividade ao processo de release. Até o presente momento foram feitos dois estudos que contribuíram para 
evolução da ferramenta e melhorias implementadas. A nova versão remove a necessidade de operações manuais, conferindo aos usuários maior agilidade na busca de pacotes (patches) de atualização e correções de software de projetos Android. Neste artigo é apresentado um estudo baseado na nova versão da ferramenta, onde foi realizado uma avaliação do impacto da ferramenta proposta no processo de gestão dos projetos do Sidia. Apresentamos uma análise qualitativa comparando os resultados com a versão anterior da ferramenta. Os resultados apresentam importante contribuição nas atividades de gestão e indicam melhorias que poderão facilitar ainda mais as atividades (Barbosa et al. 2019).

Este trabalho está organizado da seguinte maneira: a Seção 2 apresenta os desafios da melhoria de processo no DDS, o contexto onde a solução proposta foi aplicada e apresenta uma visão geral da ferramenta assim como sua evolução; a Seção 3 apresenta uma análise comparativa com os resultados da pesquisa qualitativa das duas versões da ferramenta. A Seção 4 apresenta as considerações finais e perspectivas de trabalhos futuros.

\section{MELHORIA DE PROCESSO NO DESENVOLVIMENTO DISTRIBUÍDO DE SOFTWARE}

Apesar dos avanços em todas as áreas da tecnologia, a indústria de software ainda está lutando com o desafio de desenvolver aplicativos de software que atendam a pressão de tempo, restrições de orçamento e qualidade (Pearson, McCahone e Hightower, 1995). Por conta disso, a indústria e a academia têm buscado alternativas para responder com maior rapidez na construção de novas tecnologias. Neste sentido, o Desenvolvimento Distribuído de Software (DDS) tem se tornado um elemento estratégico para garantir maior competitividade. O cenário no qual o DDS é feito, onde equipes trabalham juntas, mas não estão localizados geograficamente no mesmo ambiente, permitiu o crescimento desta área devido a economia de recursos e redução de custos (Audy e Prikladnick, 2007).

Contudo, percebeu-se que a melhoria do processo de software se torna um dos maiores desafios para o DDS (Khan, 2016). E as empresas de desenvolvimento de software reconheceram que o principal motivo da baixa qualidade do software está na falha em organizar o processo de desenvolvimento de maneira eficiente (Unterkalmsteiner, 2012). E de acordo com os resultados apresentados pelos estudos de Weimann et al (2013), a falta de ferramentas, para atividades intermediárias, pode influenciar no desempenho dos desenvolvedores.

Outro fator que corrobora com necessidade de utilizar ferramentas de suporte ao DDS, é que atualmente, o mundo está enfrentando uma crise de saúde global que leva a uma ruptura social e econômica causada pela pandemia de Covid-19 (UNDP, 2020). As organizações estão enfrentando novos desafios e são forçadas a mudar suas formas de trabalhar e interagir entre funcionários. Gerentes e membros da equipe são forçados a adaptar sua forma de trabalho, interagindo remotamente em resposta às restrições impostas pela pandemia (Korolov, 2020). Portanto, todas as organizações precisam ser ágeis para responder a este novo desafio e encontrar novas formas de atender as demandas de mercado (Badiale, 2020).

Diante desse contexto, este artigo apresenta a experiência do resultado da melhoria de uma ferramenta para automatizar uma atividade específica do time de desenvolvimento do Sidia. O propósito foi avaliar o impacto da realização da atividade com o uso da ferramenta comparado a sua versão anterior. A próxima Seção apresenta o contexto, a motivação e uma visão geral das duas versões da ferramenta chamada CLCATCH.

\subsection{A Ferramenta CLCATCH}

O produto entregue pelo Sidia consiste em uma release de atualização do sistema operacional Android personalizado para cada modelo de dispositivo móvel comercializado pela Samsung. Para atender essa demanda os projetos são divididos em desenvolvimento e atualização de S.O. para dispositivos já lançados no mercado e novos modelos. As atualizações de S.O. podem incluir novos requisitos de clientes ou pacotes de aprimoramento de segurança, que são um conjunto de pacotes contendo funcionalidades para melhoria do S.O. Cada pacote é armazenado em um sistema de controle de versão, de acordo com cada modelo de dispositivo que são enviados em lotes, chamados de patches. O uso de pacotes, ou patches, visa a otimização do processo de desenvolvimento de software, uma vez que este ciclo de desenvolvimento é feito em conjunto com outros institutos de $\mathrm{P} \& \mathrm{D}$, distribuídos geograficamente em diversos países localizados na América Latina e Ásia (Barbosa, 2009). 
O processo de release é mantido por uma equipe específica, formada por engenheiros de software que ocupam o papel de líder de projeto (LP). Esta equipe é responsável pela gestão das demandas de cada projeto, tendo como atividades principais a inclusão das atualizações de segurança e o monitoramento de novos requisitos das operadoras de telefonia. As demandas de desenvolvimento são solicitadas pelas operadoras ou pelo Google, por meio de novos requisitos de software (Barbosa, 2019).

Para melhor organização dos times distribuídos, o Sidia emprega uma abordagem que utiliza pacotes (patches) de atualização, chamadas de Propagation Patch (PP). E para criar uma nova release, o time de LPs precisa compilar implementações e novas solicitações e aplicar as mudanças para versão de S.O. em cada modelo, gerando modificações que são agrupadas em patches, integradas dentro de cada PP. A vantagem da utilização de PPs é o aproveitamento de código, que pode ser aplicado em diferentes modelos com a mesma arquitetura de hardware (Barbosa, 2019).

O LP é o responsável pela aplicação de novos requisitos, atualmente utiliza a ferramenta CLCatch para obter os pacotes de atualização que precisam ser aplicados na release, o usuário precisa realizar o upload do relatório de falhas na página web da ferramenta e aguardar o resultado ser apresentado na tela. Contudo, essa busca é feita através da leitura das descrições dos pacotes de mudanças no controlador de versões, ocasionando nesse processo um overhead no início ao fazer o upload do relatório e durante a busca dos pacotes, mesmo sendo muito mais rápido e seguro, comparado a forma manual, ainda assim existem pontos de melhoria a serem explorados.

Para auxiliar no processo busca dos pacotes a ferramenta CLCatch foi desenvolvida, conforme vista na Figura 1.

\section{CLCATCH}

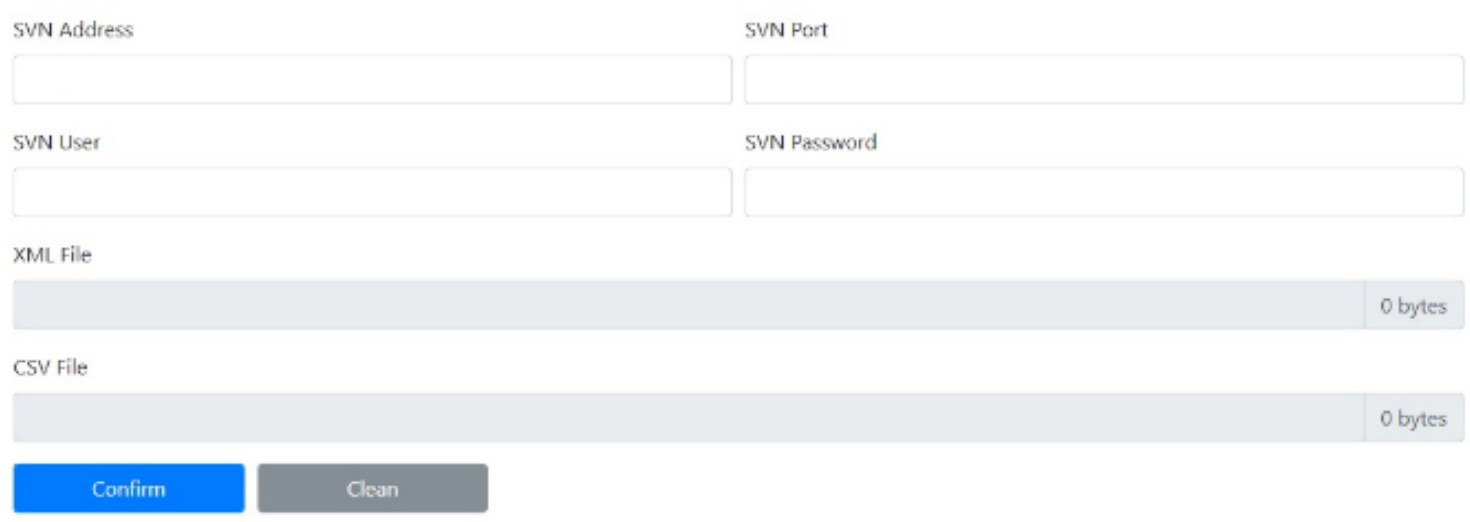

Figura 1. Visão Geral da Ferramenta CLCATCH (Barbosa, 2019)

A primeira versão da ferramenta CLCATCH é uma aplicação web, desenvolvida com as linguagens de programação Java e PHP, possui seis campos de entrada sendo cinco deles obrigatórios e somente o campo o campo CSV file é opcional. Todas essas entradas de dados são necessárias para que a aplicação possa se conectar com o controlador de versão e buscar as atualizações de segurança necessários para aplicar na release.

Com base solicitações recebidas na pesquisa qualitativa de Barbosa et al (2019), foi desenvolvido uma nova versão ferramenta implementada na plataforma do navegador Chrome. A escolha da tecnologia foi decidida com base nos pontos de melhoria sugeridos na primeira versão da ferramenta e após avaliar os meios de como atender as solicitações, chegou-se a proposta de desenvolver uma aplicação Extention do navegador Google Chrome, pois dessa forma o usuário não precisaria dar entrada em qualquer tipo de dado ou configuração na tela (Figura 2). 


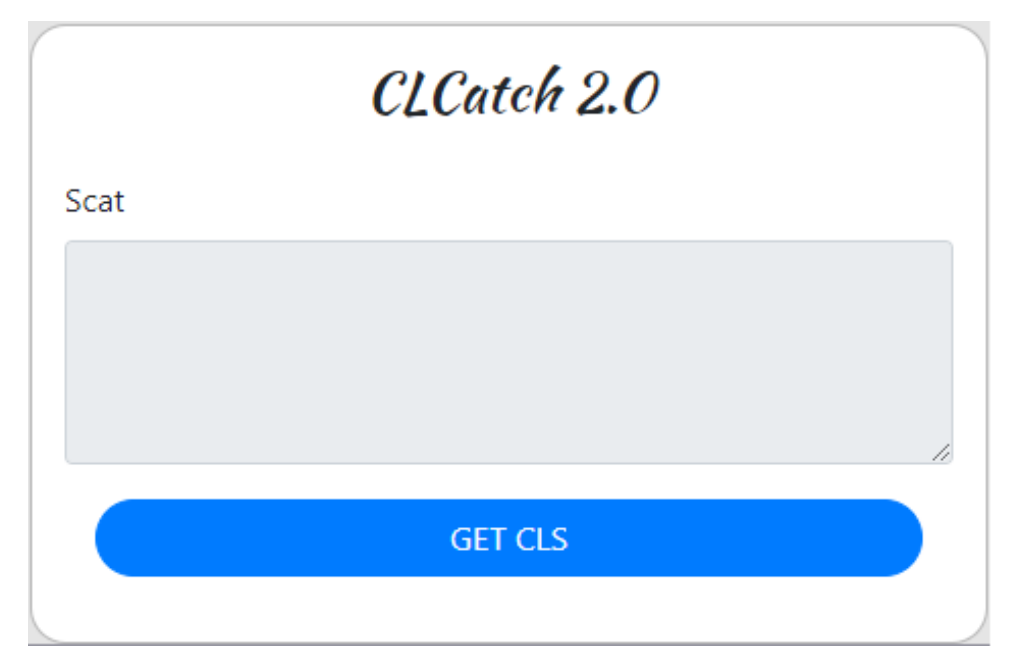

Figura 2. Visão da Ferramenta CLCATCH 2.0

A ferramenta CLCatch 2.0 foi desenvolvida utilizando as tecnologias JavaScript, jQuery e Bootstrap, tem uma interface muito simples comparado a sua versão anterior, para executar o usuário precisa estar com a página de relatório de falhas aberta em uma aba do Chrome, com apenas um clique no botão GET CLS e o resultado é apresentado na caixa de texto. Esse objetivo foi alcançado através do uso de uma API, a qual permite realização de uma chamada a partir do Extension do Chrome passando os parâmetros necessários efetuar para busca das atualizações de segurança. Os parâmetros necessários são capturados pelo CLCatch 2.0 da página web do relatório de falhas que está carregado na aba do Chrome. Ao reunir todos os parâmetros necessários a aplicação realiza uma chamada para a API que por sua vez retorna os dados e estes são apresentados na tela.

\section{RESULTADOS QUALITATIVOS}

A análise foi importante para identificar pontos de melhoria e avaliar a percepção da equipe, sobre o uso da ferramenta CLCATCH na sua versão 1.0 e posteriormente na 2.0. A amostra foi escolhida aleatoriamente, e os participantes realizaram a aceitação e caracterização do estudo, por meio de formulário no Google Forms. A Tabela 1 apresenta os resultados do questionário TAM aplicado e em seguida os gráficos apresentam os resultados do questionário TAM seguido de suas respectivas análises comparativas dos resultados qualitativos entre as duas versões da ferramenta. Além das perguntas listadas na Tabela 1, foram incluídas duas perguntas descritivas e opcionais, onde o LP poderia registrar quais as outras ferramentas usadas para a busca de pacotes e um campo para sugestões, os resultados das respostas descritivas serão utilizados como exemplos no decorrer da análise dos resultados. A pesquisa foi realizada com 16 LPs, os participantes usaram a ferramenta em média durante um mês.

Para facilitar a demonstração da análise qualitativa, foram plotados os gráficos referentes aos resultados da primeira pesquisa ao lado dos resultados da pesquisa atual. Os dados estão agrupados em facilidade de uso, desempenho e utilidade, algumas perguntas não são equivalentes, portanto, não será possível uma comparação direta, somente uma análise isolada dos seus resultados. 
Tabela 1. Avaliação qualitativa dos participantes do estudo

\begin{tabular}{|c|c|c|c|c|c|c|c|c|}
\hline Métrica & 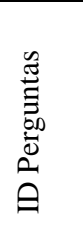 & Itens de Verificação & 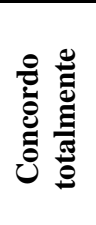 & 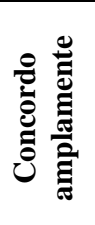 & 冚 & 莺 & 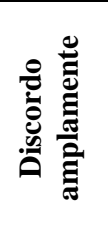 & 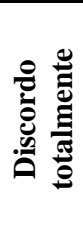 \\
\hline \multirow{4}{*}{ 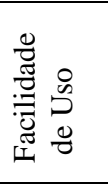 } & Q1 & Foi fácil de aprender a usar a CLCATCH 2.0 & 12 & 3 & 1 & 0 & 0 & 0 \\
\hline & Q2 & Consegui usar a CLCATCH 2.0 da forma que eu queria & 14 & 2 & 0 & 0 & 0 & 0 \\
\hline & Q3 & $\begin{array}{l}\text { Eu entendia o que acontecia na minha interação com a } \\
\text { CLCATCH } 2.0\end{array}$ & 9 & 5 & 2 & 0 & 0 & 0 \\
\hline & Q4 & É fácil ganhar habilidade no uso da CLCATCH 2.0 & 11 & 5 & 0 & 0 & 0 & 0 \\
\hline \multirow{4}{*}{ 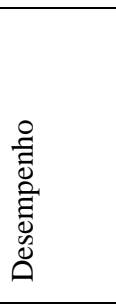 } & Q5 & $\begin{array}{l}\text { A ferramenta me permitiu realizar as buscas de forma } \\
\text { mais rápida do que utilizando a versão anterior }\end{array}$ & 10 & 5 & 1 & 0 & 0 & 0 \\
\hline & Q6 & $\begin{array}{l}\text { Considero que a CLCATCH } 2.0 \text { no formato Extension } \\
\text { do Chrome melhorou a minha atividade no processo de } \\
\text { geração de releases }\end{array}$ & 9 & 5 & 2 & 0 & 0 & 0 \\
\hline & Q7 & Consegui buscar facilmente os pacotes que eu desejava & 9 & 7 & 0 & 0 & 0 & 0 \\
\hline & Q8 & $\begin{array}{l}\text { CLCATCH } 2.0 \text { melhora minha produtividade na busca } \\
\text { por pacotes }\end{array}$ & 13 & 2 & 1 & 0 & 0 & 0 \\
\hline \multirow{4}{*}{$\frac{\mathscr{m}}{\frac{\pi}{\pi}}$} & Q9 & Considero a CLCATCH 2.0 útil & 11 & 5 & & 0 & 0 & 0 \\
\hline & Q10 & Preciso de outras ferramentas para buscar os pacotes & 1 & 0 & 10 & 2 & 2 & 1 \\
\hline & Q11 & $\begin{array}{l}\text { A CLCATCH } 2.0 \text { aprimora a busca segura de pacotes } \\
\text { em relação ao processo anteriormente utilizado }\end{array}$ & 9 & 7 & 0 & 0 & 0 & 0 \\
\hline & Q12 & $\begin{array}{l}\text { A CLCATCH } 2.0 \text { melhora o processo de geração de } \\
\text { releases }\end{array}$ & 10 & 5 & 1 & 0 & 0 & 0 \\
\hline
\end{tabular}

Com relação facilidade de uso, a Figura 3, demonstra que houve um expressivo incremento positivo nos números acerca das perguntas relacionadas usabilidade, visto que no geral, foi obtido um aumento de $36 \%$ no percentual de respostas "Concordo Totalmente". Analisando os resultados individualmente, pode ser notado que os maiores ganhos foram:

- Na facilidade em aprender a usar a ferramenta: isso pode ser explicado pela facilidade com a qual a ferramenta é utilizada, com apenas um clique em um botão os resultados são apresentados na tela do usuário;

- Ao utilizar a ferramenta da forma que usuário queria: esse item pode ser percebido como um resultado positivo em resposta ao atendimento a solicitação da primeira pesquisa a qual solicitava a remoção da necessidade da execução do upload do relatório de falhas na página web da ferramenta.
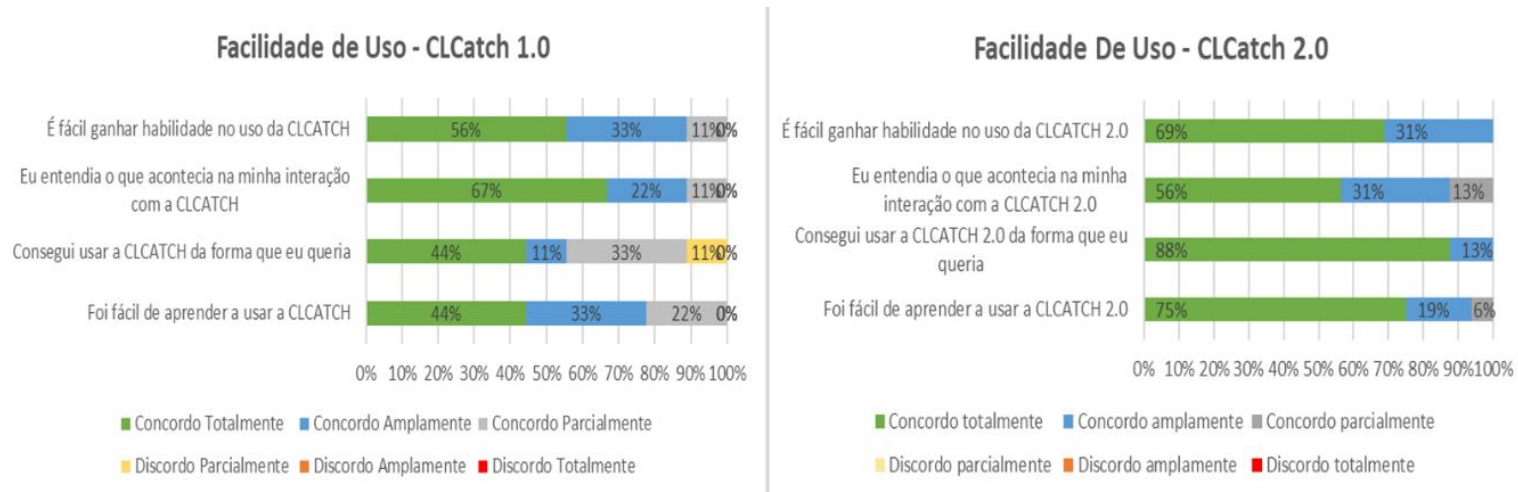

Figura 3. Facilidade de Uso

No que diz respeito a percepção de desempenho, representado na Figura 4, a pergunta que teve o melhor desempenho foi em relação ao ganho de produtividade com um aumento de $14 \%$ em comparação com a versão anterior. Contudo, de modo geral nota-se pouca variação nas outras perguntas, houve uma leve migração dos percentuais das respostas "Concordo Parcialmente" e "Concordo Totalmente" para "Concordo Amplamente". 
É possível que os ganhos com o desempenho não estejam sendo percebidos porque as duas versões automatizam o processo que inicialmente era feito de forma manual.

Em relação a melhoria das atividades com base formato da ferramenta como extension do Chrome, trata-se de uma pergunta que não existia no formulário anterior, portanto foi conduzido uma análise isolada, na qual foi identificado que os participantes que responderam "Concordo Amplamente" e "Concordo Parcialmente", demandaram um requisito em comum "Tentar implementar a busca de pacotes de CP", é importante observar que esse é um requisito novo quem também não é suportado pela versão anterior da ferramenta, no entanto isso demonstra uma oportunidade de melhoria que poderá agregar maior produtividade ao processo.

Outro ponto a observar é que a percepção de melhoria em relação a versao 1.0 e a versão 2.0 , foi menor do que em comparação ao estado inicial onde era manual com a versão 1.0, apesar disso teve um resultado melhor com o CLCatch 2.0 quando somado as repostas "Concordo Totalmente" e "Concordo Amplamente", isso indica uma leve aumento percepção de ganho de velocidade nos resultados.

\section{Desempenho - CLCatch 1.0}

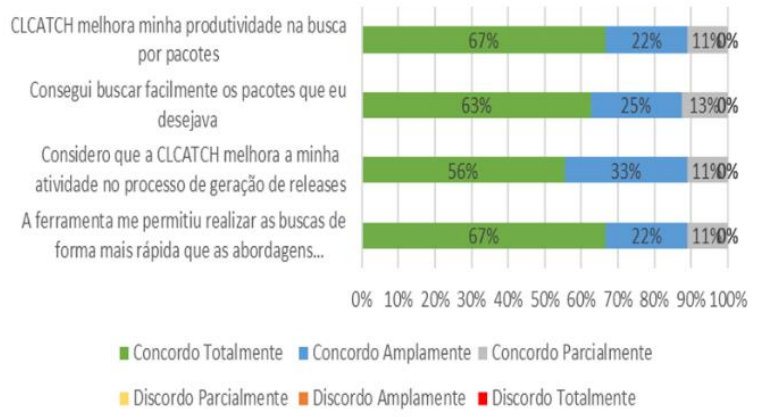

Desempenho - CLCatch 2.0

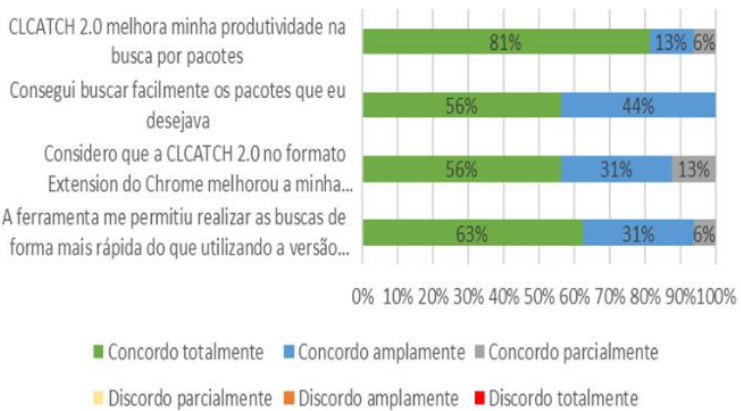

Figura 4. Desempenho

No que diz respeito a percepção dos LPs sobre a utilidade, a Figura 5 demonstra um incremento significativo na melhoria do processo de geração de releases, com um aumento de $20,5 \%$ quando somado as respostas "Concordo Totalmente" e "Concordo Amplamente", o resultado sugere que ferramenta agrega maior produtividade ao processo comparado a sua versão anterior. Outro item identificado com expressiva melhoria está relacionado ao aprimoramento da confiabilidade dos resultados das buscas dos pacotes, isso se explica pelo fato de a nova ferramenta utilizar a base de dados oficial da Samsung.

A pergunta sobre a utilização de outras ferramentas com a mesma finalidade foi incluída nessa pesquisa com o objetivo de identificar quais ferramentas estão sendo usadas para uma posterior análise dos requisitos que podem ser agregados ao CLCatch 2.0. Como se trata de uma pergunta que não existia na pesquisa anterior, nessa avalição é realizado uma análise individual e não comparativa. Essa foi a questão que levantou maior variabilidade nos resultados, a maioria dos LPs responderam que a ferramenta não busca um tipo específico de pacotes, o que revela uma oportunidade de melhoria na interface da aplicação para demonstrar que é possível buscas específicas, uma sugestão enviada por um LP foi a seguinte "Adicionar um botão de copy; Mostrar CLs AP/CP/CSC em quadros separados", aplicando esses requisitos é possível atender a demanda da maioria das sugestões reportadas na pesquisa. 

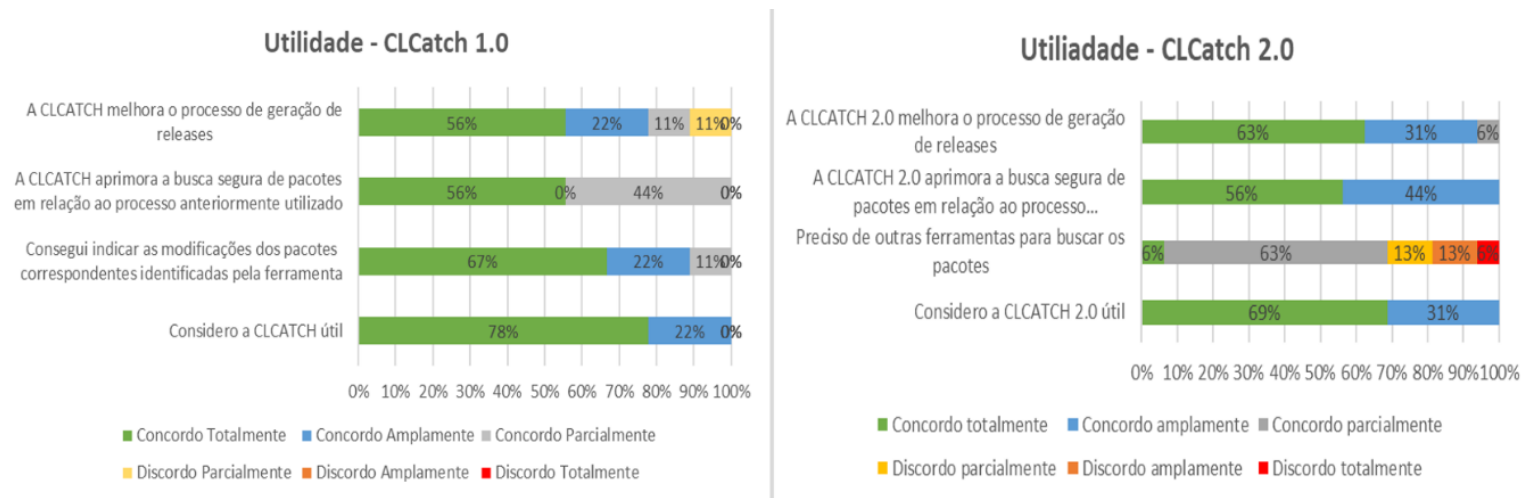

Figura 5. Utilidade

O estudo qualitativo foi útil pois contribuiu para a homologação da ferramenta oficialmente para atividade de buscas de pacotes para geração de releases de segurança e permitiu a identificação de novos requisitos como os seguintes: 1) Buscar pacotes de CP; 2) Adicionar um botão para copiar os resultados; 3) Classificar os tipos de pacotes em campos separados; 4) Integrar a ferramenta para disparar as builds na ferramenta de integração contínua; 5) Manter as configurações do binário de referência para agilizar a próxima execução.

\section{CONCLUSÃO}

Este artigo é um relato de experiência que apresenta uma análise qualitativa dos resultados da evolução e melhoria de uma ferramenta de automação de atividade no processo de release. É um estudo de extensão derivado da primeira publicação em Barbosa et al (2019), na qual os resultados iniciais foram descritos detalhadamente através da avaliação dos resultados quantitativos e qualitativos sobre as dificuldades enfrentadas pelos PLs durante o processo de gerenciamento de release, especificamente na etapa de coleta dos pacotes de correções.

No trabalho anterior, realizamos um estudo empírico com a participação de 9 LPs, utilizamos um questionário online com perguntas baseadas no TAM, considerando facilidade de uso, desempenho e utilidade. Com base nos resultados obtidos, foram identificadas algumas oportunidades de melhorias, as quais foram usadas como base para o desenvolvimento de uma nova versão da ferramenta e uma nova pesquisa dessa vez com 16 LP's participantes.

Observou-se que a maioria dos problemas identificados na primeira versão da CLCatch, ocorreram no grupo de perguntas sobre facilidade de uso e utilidade. Como melhoria, uma nova versão ferramenta foi desenvolvida e disponibilizada na própria página web onde o relatório de falhas é reportado, além disso, com base nas sugestões do estudo anterior foi removido a necessidade de fazer o download do relatório e upload na ferramenta, com isso os indicadores de facilidade de uso e utilidade tiveram um expressivo aumento com o CLCatch 2.0. A pesquisa qualitativa foi essencial para a identificação da percepção dos usuários em cada item pesquisado e coletar mais sugestões para o próximo ciclo de melhoria.

A publicação de informações do setor privado é uma atividade desafiadora devido as restrições com dados sensíveis a segurança da informação, contudo, para que seja possível a troca de conhecimento, espera-se incentivar os profissionais da indústria de desenvolvimento de softwares para dispositivos móveis a compartilhar suas experiências no gerenciamento do processo de releases para dispositivos móveis.

Com base nesses resultados, um novo conjunto de requisitos será projetado e implementados para atender as sugestões dos LPs a fim de contribuir positivamente com os resultados com o processo de gerenciamento de release. Dessa forma novos estudos serão aplicados no qual será possível traçar a curva de ganho em todas as etapas de melhoria do processo. 


\section{AGRADECIMENTOS}

Este trabalho teve suporte parcial da Samsung Eletronica da Amazonia Ltda sob o auspício da lei de informática $\mathrm{N}^{\mathrm{o}} 8.387 / 91$.

\section{REFERÊNCIAS}

Audy, Joge. Prikladnick, Rafael, 2007. Desenvolvimento Distribuído de Software - Desenvolvimento de Software com Equipes Distribuídas. Série Campus. Elsevier Editora, Rio de Janeiro, Brasil.

Badiale, Maria Eugenia. 2020. The dynamics of communication in global virtual software development teams - A case study in the agile context during the Covid-19 pandemic. Department of Business Studies Uppsala University, 2020.

Barbosa, H. O. et all. 2019. Uma Análise do uso de Ferramentas em Desenvolvimento Distribuído de Software para Atualização da Plataforma Android. 2019. IADIS Americana WWW/Internet 2019, pp. 29- 38. Portugal.

Barbosa, H. O. et all. 2020. Developing a release management tool to support global software development: an experience report on Android platform. In Proceedings of the 15th International Conference on Global Software Engineering (ICGSE '20). Association for Computing Machinery, New York, NY, USA, 117-121.

Khan. A. A. 2016. A framework for assisting software process improvement program in global software development: student research abstract. In Proceedings of the 31st Annual ACM Symposium on Applied Computing (SAC '16). Association for Computing Machinery, New York, NY, USA, 1580-1581.

Korolov, M., 2020. Remote Agile Development: Top Tips and Tools For Managing Dispersed Teams. CIO. Available at: https://www.cio.com/article/3538490/remote-agile-development-top-tips-and-tools-for-managing-dispersedteams.html. Acessado 20 Abril 2020.

Pearson, J. M., McCahon, C. S., \& Hightower, R. T. (1995). Total quality management: are information system mangers ready? Information \& Management, 29, 252-63.

Lima, A. Costa, B. França, 2016, Gerência Flexível de Processos de Software com o Ambiente WebAPSEE. XIII Sessão de Ferramentas-XX SBES.

Sangwan, R. et all. Global Software Development Handbook. Auerbach Publications, 2007.

UNDP. 2020. Coronavirus Disease COVID-19 Pandemic | UNDP. Available at: https://www.undp.org/content/undp/en/home/coronavirus.html. Acessado em 15 outubro 2020.

Unterkalmsteiner, M, et all. 2012. Evaluation and measurement of software process improvement - a systematic literature review. IEEE Transactions on Software Engineering. 2012.

Weimann, M. P. et al. 2013 Enhancing team performance through tool use: How critical technology related issues influence the performance of virtual project teams. IEEEE Transactions on Professionals Communication. 